\title{
Vulnerabilidad de la población de Guerrero, México, ante las epidemias de COVID-19 y dengue
}

\section{Vulnerability of the population of Guerrero state, Mexico, to COVID-19 and dengue epidemics.}

\author{
Norma Ivone Peña-Galeana ${ }^{1}$ \\ Universidad Autónoma de Guerrero, México \\ Ricardo Peña-Galeana ${ }^{2}$ \\ Universidad Autónoma de Guerrero, México \\ Pedro Vidal Tello-Almaguer ${ }^{3}$ \\ Universidad Autónoma de Guerrero, México
}

\begin{abstract}
Resumen
El presente artículo analiza la situación del COVID-19 y el Dengue en los municipios del estado de Guerrero (en México), así como los grupos poblacionales afectados clasificados por edad y sexo. Se analiza también la situación de los factores asociados con las medidas de prevención y control de ambas epidemias, incluyendo entre estos: la disponibilidad de agua entubada, la suficiencia alimentaria de menores y adultos, y la afiliación a servicios de salud. Asimismo, con base en los factores antes mencionados, se realiza una clasificación de los municipios de Guerrero según el nivel de vulnerabilidad de su población ante ambas epidemias en conjunto (vulnerabilidad entendida como la susceptibilidad de la población a ser afectada por Dengue o COVID-19, dada la situación de los factores en estudio).
\end{abstract}

Palabras clave: Dengue, COVID-19, Vulnerabilidad de la población, Población en riesgo.

1 Maestra en ingeniería. Unidad Académica de Ciencias y Tecnologías de la Información, Universidad Autónoma de Guerrero, México. Correo-e: normaivone@yahoo.com iD https://orcid.org/ 0000-0003-3827-7817.

2 Maestro en ingeniería. Unidad Académica de Ciencias y Tecnologías de la Información, Universidad Autónoma de Guerrero, México. Correo-e: rpgaleana@msn.com iD https://orcid.org/0000-0002-5048-7799.

3 Doctor en Geografía. Centro de Investigación y Posgrado en Estudios Socioterritoriales, Universidad Autónoma de Guerrero, México. Correo-e: pvidalt@hotmail.com (DD https://orcid.org/0000-0003-0473-3562. 
Norma Ivone Peña-Galeana, Ricardo Peña-Galeana, Pedro Vidal Tello-Almague Vulnerability of the population of Guerrero state, Mexico, to COVID-19 and dengue epidemics.

\begin{abstract}
The present article analyzes the situation of COVID-19 and dengue in the municipalities of the state of Guerrero, Mexico, as well as the situation of affected population groups classified by age and sex. Factors associated with prevention and control measures of both epidemics are also analyzed, including: availability of potable piped water, food sufficiency for minors and adults, and affiliation to health care services. Likewise, based on the aforementioned factors, a classification of the municipalities comprising the state of Guerrero is made according to the population's level of vulnerability to both epidemics as a whole. Vulnerability is defined as the susceptibility of the population to be affected by COVID-19 and dengue given the situation of the studied factors.
\end{abstract}

Keywords: Dengue; COVID-19; Population vulnerability; Population at risk.

\title{
Introducción
}

El 30 de enero de 2020, la Organización Mundial de la Salud (OMS) declaró a la enfermedad COVID-19 como una emergencia internacional y, para el 11 de marzo, el brote de COVID-19 fue declarado como una pandemia (OMS, 2020a; OMS, 2020b). En México se confirmó el primer caso de COVID-19 el 28 de febrero, pero fue hasta el 15 de marzo que se registró el primer caso confirmado en el estado de Guerrero, ubicado al sur del país (Gobierno de México, 2020a; Gobierno del estado de Guerrero, 2020a).

El estado de Guerrero, está clasificado como una de las entidades federativas más afectadas por la epidemia de COVID-19 en México, situación que puede agravarse aún más con la llegada de la temporada de dengue que se registra entre los meses de mayo a noviembre cada año, lo que implica que la población guerrerense deberá hacer frente a dos padecimientos virales al mismo tiempo.

Dado que el COVID-19 presenta síntomas y medios de transmisión similares a los de la gripe estacional, se espera que esta epidemia evolucione con una disminución de casos durante el verano y una reemergencia en los meses de invierno; sin embargo, con el fin de la cuarentena y la reactivación de la actividad económica aumentará el desplazamiento poblacional, lo que se prevé que en el corto plazo se traduzca en un incremento en los casos de COVID-19 en todos los estados y municipios del país.

El estado de Guerrero, por tanto, enfrentará la reanudación de las actividades económicas con una tendencia creciente en el número de casos de COVID-19, a lo cual es necesario añadir el inicio de la temporada de dengue (que en Guerrero se registra durante los meses de mayo-noviembre) con una propensión creciente en el número de casos a nivel estatal, tomando en cuenta que la entidad es una de las que registra más casos de dengue en el contexto nacional y que el número de casos de dengue a nivel mundial va en ascenso (OMS, 2020c). 
Lo anterior, hace necesario evaluar el nivel de vulnerabilidad la población afectada por el COVID-19 y el dengue, identificando sus características y analizando la posible interacción entre las medidas que se establecen para la prevención y el control ambas enfermedades y su efecto sobre la población guerrerense.

\section{Área de estudio}

Como puede observarse en la Figura 1, el estado de Guerrero se ubica al sur de México, en la costa del Océano Pacífico, se divide en 81 municipios y limita al noroeste con el estado de Michoacán, al norte con los estados de México y Morelos, al noreste con el estado de Puebla, al este con el estado de Oaxaca y al sur, con el Océano Pacífico.

Figura 1. Localización del área de estudio: Estado de Guerrero, México.

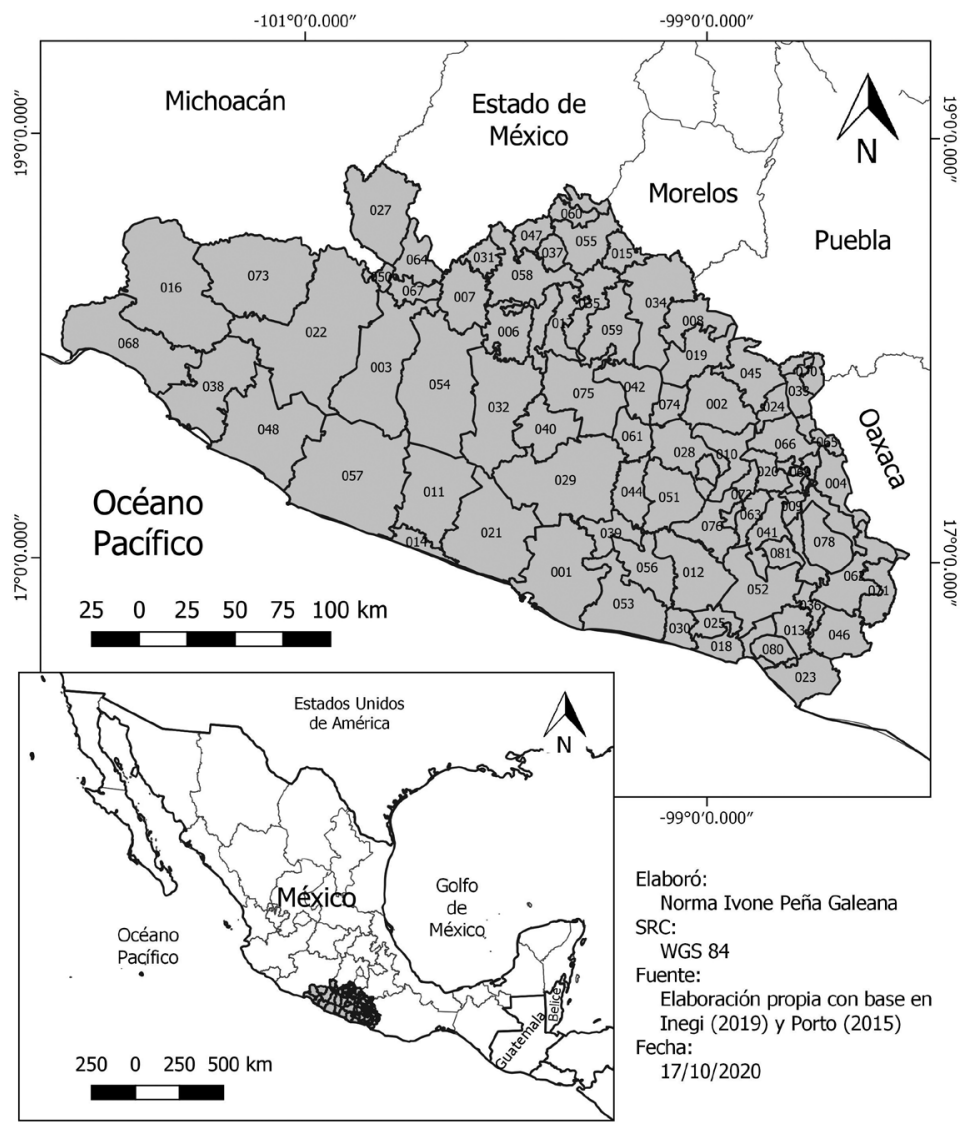

Fuente: Elaboración propia con base en la cartografía del Instituto de Estadística, Geografía e Informática de México - Inegi (2019) y Porto (2015). 
Guerrero tiene una superficie de $63794 \mathrm{~km} 2$ que representa el 3,3\% del país y un litoral de aproximadamente $500 \mathrm{~km}$ de largo. Su capital es la ciudad de Chilpancingo de los Bravo, situada en el centro de la entidad.

Tabla 1. Municipios del estado de Guerrero, México, agrupados por jurisdicciones sanitarias.

\begin{tabular}{|c|c|c|}
\hline Norte & Montaña & Costa Chica \\
\hline $\begin{array}{c}\text { 006 Apaxtla } \\
\text { 008 Atenango del Río } \\
\text { 015 Buenavista de Cuéllar } \\
\text { 017 Cocula } \\
\text { 019 Copalillo } \\
\text { 026 Cuetzala del Progreso } \\
\text { 031 General Canuto A. Neri } \\
\text { 034 Huitzuco de los Figueroa } \\
\text { 035 Iguala de la Independencia } \\
\text { 037 Ixcateopan de Cuauhtémoc } \\
\text { 047 Pedro Ascencio Alquisiras } \\
\text { 049 Pilcaya } \\
\text { 055 Taxco de Alarcón } \\
\text { 058 Teloloapan } \\
\text { 059 Tepecoacuilco de Trujano } \\
\text { 060 Tetipac }\end{array}$ & $\begin{array}{c}004 \text { Alcozauca de Guerrero } \\
\text { 005 Alpoyeca } \\
\text { 009 Atlamajalcingo del Monte } \\
\text { 010 Atlixtac } \\
\text { 020 Copanatoyac } \\
\text { 024 Cualac } \\
\text { 033 Huamuxtitlán } \\
\text { 041 Malinaltepec } \\
\text { 043 Metlatónoc } \\
\text { 045 Olinalá } \\
\text { 063 Tlacoapa } \\
\text { 065 Tlalixtaquilla de Maldonado } \\
\text { 066 Tlapa de Comonfort } \\
\text { 069 Xalpatláhuac } \\
\text { 070 Xochihuehuetlán } \\
\text { 072 Zapotitlán Tablas } \\
\text { 076 Acatepec } \\
\text { 078 Cochoapa el Grande } \\
\text { 081 Iliatenco }\end{array}$ & $\begin{array}{c}012 \text { Ayutla de los Libres } \\
\text { 013 Azoyú } \\
\text { 018 Copala } \\
\text { 023 Cuajinicuilapa } \\
\text { 025 Cuautepec } \\
\text { 030 Florencio Villarreal } \\
\text { 036 Igualapa } \\
\text { 046 Omepetec } \\
\text { 052 San Luis Acatlán } \\
\text { 053 San Marcos } \\
\text { 056 Tecoanapa } \\
\text { 062 Tlacoachistlahuaca } \\
\text { 071 Xochistlahuaca } \\
\text { 077 Marquelia } \\
\text { 080 Juchitán }\end{array}$ \\
\hline Tierra Caliente & Costa Grande & Centro \\
\hline $\begin{array}{c}\text { 003 Ajuchitlán del Progreso } \\
\text { 007 Arcelia } \\
\text { 022 Coyuca de Catalán } \\
\text { 027 Cutzamala de Pinzón } \\
\text { 050 Pungarabato } \\
\text { 054 San Miguel Totolapan } \\
\text { 064 Tlalchapa } \\
\text { 067 Tlapehuala } \\
\text { 073 Zirándaro }\end{array}$ & $\begin{array}{c}\text { 011 Atoyac de Álvarez } \\
\text { 014 Benito Juárez } \\
\text { 016 Coahuayutla de José Ma. } \\
\text { Izazaga } \\
\text { 021 Coyuca de Benítez } \\
\text { 038 José Azueta } \\
\text { 048 Petatlán } \\
\text { 057 Técpan de Galeana } \\
\text { 068 La Unión de Isidoro Montes } \\
\text { de Oca }\end{array}$ & $\begin{array}{c}\text { 002 Ahuacuotzingo } \\
\text { 028 Chilapa de Álvarez } \\
\text { 029 Chilpancingo de los Bravo } \\
\text { 032 General Heliodoro Castillo } \\
\text { 039 Juan R. Escudero } \\
\text { 040 Leonardo Bravo } \\
\text { 042 Mártir de Cuilapan } \\
\text { 044 Mochitlán } \\
\text { 051 Quechultenango } \\
\text { 061 Tixtla de Guerrero } \\
\text { 074 Zitlala } \\
\text { 075 Eduardo Neri } \\
\text { 079 José Joaquín de Herrera }\end{array}$ \\
\hline \multicolumn{3}{|l|}{ Acapulco } \\
\hline 001 Acapulco de Juárez & & \\
\hline
\end{tabular}

Fuente: Elaboración propia utilizando las claves municipales del Instituto Nacional de Estadística, Geografía e Informática de México (Inegi, 2019) 


\section{Marco teórico-conceptual \\ El concepto de Vulnerabilidad}

El concepto de vulnerabilidad, usado con más frecuencia en las ciencias sociales, hace referencia a la posibilidad de que una población sufra daños debido a la exposición a factores de riesgo internos y externos, lo cual se ve influenciado por su capacidad para enfrentar dichos factores, así como por su habilidad para adaptarse a los cambios que esto produce (CEPAL-ECLAC, 2002; Cardona, 2003).

Este concepto de vulnerabilidad es frecuentemente relacionado con factores naturales que pueden presentar un peligro para la población, por lo que se asocia con desastres naturales causados por fenómenos naturales hidrometereológicos, inundaciones, deslizamientos de tierra, terremotos y erupciones volcánicas, entre otros. Su aplicación al ámbito social, introduce también variables demográficas tales como la estructura de la población, los procesos de urbanización y los cambios en los hábitos y costumbres de la población (DNP-Colombia, 2007; Foschiatti, 2008; Saavedra, 2010).

En el área de la salud, hablar de vulnerabilidad implica hablar de la exposición de la población a algún agente patógeno que puede derivar en una enfermedad dependiendo de la influencia de factores ambientales o sociales tales como como la temperatura, la precipitación, el estado nutricional de la población o los avances en la medicina, por citar algunos (Foschiatti, 2008; Araujo, 2015).

Generalizando, podemos entonces definir la vulnerabilidad como la posibilidad de que un sistema sufra algún daño debido a la actuación de factores internos o externos, y a su propia respuesta ante dichos factores. Esta definición, por tanto, incluye tres aspectos a considerar: factores de riesgo, respuesta social y la influencia del tiempo y lugar en que estos se presentan (Shitangsu, 2014).

\section{Definiciones asociadas al Dengue y al COVID-19}

La enfermedad dengue es causada por un virus (Denguevirus, con cuatro serotipos DENV-1, DENV-2, DENV-3 y DENV-4) transmitido por un mosquito del género aedes (A. aegypti y A. albopictus). La Secretaría de Salud de México (SSA), en las Normas Oficiales NOM-032-SSA2-2002 y NOM-032-SSA2-2014 y en sus Manuales de procedimientos (SSA, 2003; SSA, 2015; SSA, 2017a), menciona que la clasificación de los casos de 
dengue se realiza con base en su severidad, y ésta va desde casos con síntomas febriles leves, conocidos como Dengue No Grave (DNG), hasta casos severos conocidos como Dengue con Signos de Alarma (DCSA) y Dengue Grave (DG), que se acompañan de dificultad respiratoria, hemorragias graves o falla orgánica que puede llevar a la muerte del paciente.

La Organización Mundial de la Salud (OMS) considera al dengue como una virosis pandemiógena, es decir, como una enfermedad causada por virus con potencial de convertirse en pandemia de rápida propagación, lo que lleva a que mantenga un monitoreo permanente de la evolución del padecimiento y de las estrategias de prevención y control del dengue en el mundo (OMS, 2020c; OMS, 2020d).

Actualmente, existe una vacuna para el dengue en el mercado, pero debido a que su eficacia aún se encuentra bajo análisis (OMS, 2016; OMS, 2018), el tratamiento de los casos se enfoca al mantenimiento del paciente mientras supera la enfermedad, lo cual puede incluir desde la permanencia del paciente en su domicilio por unos días, hasta su hospitalización entre siete y diez días.

El COVID-19, por otro lado, es una enfermedad respiratoria ocasionada por el nuevo tipo de coronavirus SARS-CoV-2, cuyo espectro va desde casos asintomáticos y pacientes con síntomas leves de resfriado, hasta cuadros graves de insuficiencia respiratoria o falla orgánica que pueden llevar a la muerte (OMS, 2020e).

Hasta el momento, no existen pruebas de que la infección por este virus produzca inmunidad, aunque se espera que esto ocurra en el corto plazo; tampoco existe una vacuna o tratamiento específico para esta enfermedad, por lo que su manejo se centra en la atención del paciente según la gravedad de sus síntomas, y en la prevención con medidas de distanciamiento social.

\section{Marco metodológico}

Para el análisis del COVID-19 y el dengue en el estado de Guerrero, se utilizaron las definiciones de la Organización Mundial de la Salud en relación con los grupos poblacionales de riesgo y los factores de prevención y riesgo coincidentes en ambos padecimientos.

La información asociada con ambas epidemias difiere en cuanto fuentes y periodos de tiempo considerados debido a que el dengue, a diferencia del COVID-19, no es una enfermedad nueva en el estado de Guerrero. 
- Así, para el análisis de la epidemia de COVID-19 se utilizaron los datos proporcionados por el Gobierno del estado de Guerrero, actualizados al 28 de mayo de 2020 (Gobierno del estado de Guerrero, 2020b), que incluyeron los casos positivos y las defunciones clasificados por municipio. Con el fin de identificar los casos positivos de COVID-19 clasificados por edad y sexo, se utilizó la información proporcionada en la plataforma "Datos abiertos de México", correspondientes a los casos de COVID-19 en el estado de Guerrero hasta el 13 de abril de 2020 (Gobierno de México, 2020b).

- $\quad$ En relación con el dengue, se trabajó con los datos proporcionados por la Organización Panamericana de la Salud (OPS), a través de la Plataforma de Información en Salud para las Américas (Plisa), que incluyeron la información de los casos confirmados de dengue en los municipios del estado de Guerrero en 2018 (OPS, n.d.). Asimismo, para el análisis de los casos confirmados agrupados por edad y sexo, se recurrió a la información proporcionada en la misma plataforma para el año 2019 (la cual no incluye información a nivel municipal). Esta información se enmarcó con los datos proporcionados por la Secretaría de Salud de México a través del Panorama Epidemiológico de Dengue para el periodo 2015 a 2019 (a la semana 52), que incluyeron el total de casos acumulados de dengue clasificados por tipo (DNG, DCSA y DG).

Para el cálculo de tasas de incidencia y porcentaje de población en riesgo, así como para el análisis de factores de riesgo, se utilizaron los datos disponibles en la Encuesta Intercensal 2015 de Instituto Nacional de Estadística, Geografía e Informática de México (Inegi) secciones 1, 7, 14 y 15, referentes a población, servicios de salud, viviendas y suficiencia alimentaria en el estado de Guerrero, que incluyeron:

- $\quad$ Población total, por grupo quinquenal de edad y sexo

- $\quad$ Porcentaje de ocupantes en viviendas sin agua entubada

- Porcentaje de población no afiliada a algún servicio de salud

- Porcentaje de hogares donde algún adulto comió solo una vez al día o dejó de comer todo un día por falta de dinero 
Norma Ivone Peña-Galeana, Ricardo Peña-Galeana, Pedro Vidal Tello-Almague Vulnerability of the population of Guerrero state, Mexico, to COVID-19 and dengue epidemics.

- $\quad$ Porcentaje de hogares con población menor de 18 años donde algún menor comió solo una vez al día o dejó de comer todo un día por falta de dinero

Para los casos positivos y confirmados de ambos padecimientos, así como para las tasas de incidencia, se utilizaron las rupturas naturales de Jenks con el fin de clasificar a los municipios del estado de Guerrero en cuatro grupos, con base en la presencia de COVID-19 y dengue. Con estos datos y utilizando cartografía proporcionada por el Inegi, con el Sistema de Referencia México ITRF2008/UTM zone 14N, se elaboraron mapas que fueron procesados mediante el software QGIS.

Dado que la OPS enfatiza la necesidad de identificar zonas prioritarias para el control de las epidemias minimizando el riesgo para la población y el personal de salud involucrado (OPS, 2020a), se decidió clasificar a los municipios del estado de Guerrero en tres niveles de vulnerabilidad: baja, media y alta.

Para realizar esta clasificación, se realizó lo siguiente:

1) Para cada factor en estudio se definieron tres intervalos, a los cuales se les asignó un valor de uno a tres utilizando el criterio "más es peor". Estos intervalos se crearon utilizando las rupturas naturales de Jenks y tomando en cuenta los valores que asume la variable en estudio en todos los municipios de Guerrero.

2) A continuación, se clasificó a cada municipio con base en estos intervalos asignándosele, en cada caso, el valor correspondiente a cada variable en estudio, y se realizó una sumatoria simple para obtener un valor global que sirviera como indicador de vulnerabilidad por municipio.

3) Finalmente, con base en el valor global obtenido para todos los municipios de Guerrero, se crearon tres intervalos iguales que, bajo el criterio "más es peor", ayudaron a clasificar el nivel de vulnerabilidad de cada municipio como bajo, medio o alto.

El nivel de vulnerabilidad de los municipios de Guerrero, se expresó en el mapa de vulnerabilidad correspondiente, que se creó utilizando el sistema de referencia y el software antes mencionados. 


\section{Resultados}

\section{Panorama del COVID-19 y el Dengue en Guerrero}

Para el 28 de mayo de 2020, existían 1583 casos positivos de COVID-19 en Guerrero, registrados en 54 de los 81 municipios que conforman el estado (Gobierno del estado de Guerrero, 2020b). Lo anterior, quiere decir que el $88,2 \%$ de la población Guerrero se encontraba con riesgo alto de contraer la enfermedad COVID-19, por residir en un municipio donde se registraron casos positivos. La tasa de incidencia en Guerrero, a la fecha, era de 44,8 casos por cada cien mil habitantes, con una tasa de letalidad que durante todo el mes de mayo osciló entre el 12,1\% y el 14,5\%.

La Figura 2, muestra la distribución de casos positivos de COVID-19 en los municipios de Guerrero, donde pueden observarse que hasta esa fecha había 27 municipios sin casos, que concentran al 11,8\% de la población estatal y que se ubican principalmente al sur y sureste del estado, y cuatro municipios (Acapulco, Chilpancingo, Iguala y Taxco) con la mayor cantidad de casos ( $82,1 \%$ del total), que concentran al $38 \%$ de la población estatal y que se ubican en una franja que recorre al estado de Norte a Sur.

Figura 2. Distribución municipal de los casos positivos de COVID-19 en el estado de Guerrero, México, al 28 de mayo de 2020

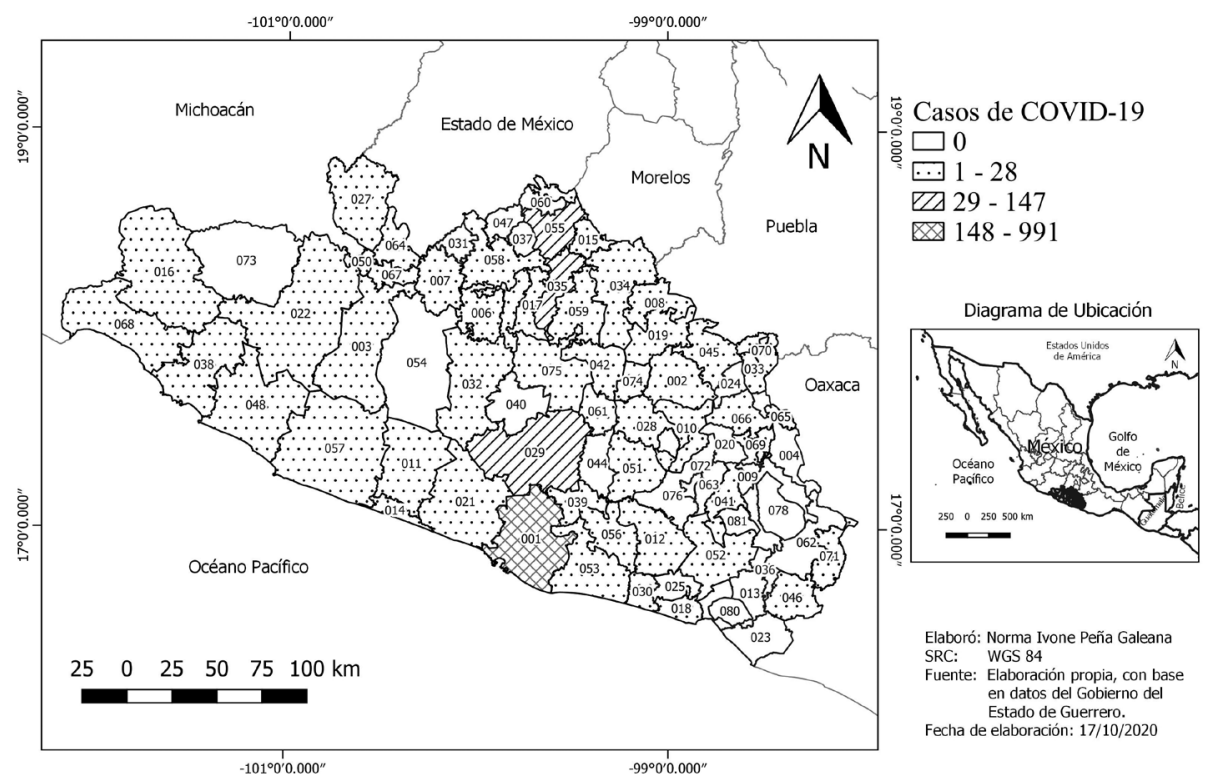

Fuente: Elaboración propia con base en la información publicada por el Gobierno del estado de Guerrero (2020b). 
Tal como se muestra en la Tabla 2, 13 municipios agrupaban al 92,9\% de los casos positivos de COVID-19, y ocho de ellos presentaban una tasa de incidencia por encima de la estatal. De entre estos municipios destacó Acapulco de Juárez, por presentar una tasa de incidencia de 122,2 casos por cada cien mil habitantes, agrupando al 62,6\% de los casos y aglutinando al 22,9\% de la población estatal. Destaca también el municipio de Xochihuehuetlán que, aunque no aparece entre los municipios con más casos, registró la tasa de incidencia más alta en Guerrero, con 180,5 casos por cada cien mil habitantes.

Tabla 2. Panorama del COVID-19 en Guerrero al 28 de mayo de 2020 (Principales municipios)

\begin{tabular}{|c|c|c|c|c|c|}
\hline Municipio & $\begin{array}{c}\text { Casos } \\
\text { positivos }\end{array}$ & Defunciones & $\begin{array}{c}\text { Tasa de } \\
\text { incidencia }\end{array}$ & $\begin{array}{c}\text { Tasa de la } \\
\text { letalidad }\end{array}$ & $\begin{array}{c}\text { de } \\
\text { población } \\
\text { estatal }\end{array}$ \\
\hline Acapulco de Juárez & 991 & 77 & 122,2 & 7,8 & $22,9 \%$ \\
\hline Chilpancingo de los Bravo & 147 & 26 & 53,8 & 17,7 & $7,7 \%$ \\
\hline Iguala de la Independencia & 111 & 45 & 73,2 & 40,5 & $4,3 \%$ \\
\hline Taxco de Alarcón & 51 & 18 & 47,0 & 35,3 & $3,1 \%$ \\
\hline Tlapa de Comonfort & 28 & 5 & 31,8 & 17,9 & $2,5 \%$ \\
\hline La Unión de Isidoro Montes de Oca & 23 & 2 & 87,0 & 8,7 & $0,7 \%$ \\
\hline Arcelia & 23 & 1 & 73,2 & 4,3 & $0,9 \%$ \\
\hline Juan R. Escudero & 20 & 1 & 80,4 & 5,0 & $0,7 \%$ \\
\hline Tixtla de Guerrero & 17 & 4 & 39,9 & 23,5 & $1,2 \%$ \\
\hline Coyuca de Benítez & 16 & 4 & 21,0 & 25,0 & $2,2 \%$ \\
\hline Pungarabato & 16 & 1 & 42,7 & 6,3 & $1,1 \%$ \\
\hline Chilapa de Álvarez & 15 & 6 & 11,6 & 40,0 & $3,7 \%$ \\
\hline Xochihuehuetlán & 13 & 5 & 180,5 & 38,5 & $0,2 \%$ \\
\hline
\end{tabular}

Fuente: Elaboración propia con base en la información publicada por el Gobierno del estado de Guerrero (2020b).

En relación con el dengue, en las últimas dos décadas se han registrado brotes reemergentes durante los cuales el estado de Guerrero ha ocupado los primeros lugares en el contexto nacional (Tabla 3). El último de estos brotes se registró en el año 2016, cuando Guerrero ocupó el primer lugar nacional con 2319 casos confirmados de dengue. Actualmente se registra un aumento del dengue en todo el mundo (OMS, 2020c), por lo que cabe esperar un aumento en el número de casos de dengue en Guerrero durante el año 2020. 
De acuerdo con el Censo de Población y Vivienda 2010 y la Encuesta Intercensal 2015 del Instituto Nacional de Estadística, Geografía e Informática (Inegi), la población total del estado de Guerrero pasó de 3’388 768 a 3'533 251 habitantes entre los años 2010 y 2015, respectivamente.

Para el año 2015, los municipios más poblados fueron Acapulco de Juárez (el único con más de 500 mil habitantes), Chilpancingo de los Bravo, Iguala de la Independencia, Chilapa de Álvarez, Zihuatanejo de Azueta, Taxco de Alarcón, Tlapa de Comonfort y Coyuca de Benítez, que agrupaban al 49,9\% de la población estatal y donde se localizan las principales ciudades de Guerrero (Acapulco, Chilpancingo, Iguala, Chilapa, Zihuatanejo, Taxco y Tlapa).

Tabla 3. Casos confirmados de dengue en el estado de Guerrero, 2015-2019

\begin{tabular}{|c|c|c|c|c|c|}
\hline \multirow{2}{*}{ Año } & \multicolumn{3}{|c|}{ Casos confirmados } & \multirow{2}{*}{$\begin{array}{c}\% \text { del total } \\
\text { nacional }\end{array}$} & \multirow{2}{*}{ Lugar nacional } \\
\hline & DNG $^{1}$ & $\mathrm{DCSA}^{2}+\mathrm{DG}^{3}$ & Total & & \\
\hline 2015 & 1208 & 522 & 1730 & $6,49 \%$ & --- \\
\hline 2016 & 1309 & 1010 & 2319 & $13,03 \%$ & 1 \\
\hline 2017 & 203 & 159 & 362 & $2,56 \%$ & 10 \\
\hline 2018 & 66 & 54 & 120 & $9,44 \%$ & 17 \\
\hline 2019 & 315 & 591 & 906 & $2,18 \%$ & 15 \\
\hline
\end{tabular}

${ }^{1}$ DNG: Dengue No Grave. ${ }^{2}$ DCSA: Dengue Con Signos De Alarma. ${ }^{3}$ DG: Dengue Grave.

Fuente: Elaboración propia con base en los datos publicados por la SSA (2017b, 2018, 2019 y 2020).

De los 81 municipios del estado, 54 registraron casos confirmados de dengue en 2018 , lo que afectó al $87 \%$ de la población guerrerense. La tasa de incidencia de dengue en 2018 fue de 22,4 casos por cada cien mil habitantes, con una tasa de letalidad de 3,7\%. Dos municipios, Acapulco de Juárez e Iguala de la Independencia, acumularon el 45\% de los casos confirmados de dengue en ese año, presentando el $32 \%$ y el $13 \%$ de los casos totales, respectivamente, y afectando, en conjunto, al $28 \%$ de la población Estatal.

La Figura 3 muestra que 26 municipios, con el 12,2\% de la población estatal, no registraron casos de dengue en 2018, mientras cuatro municipios (en orden: Acapulco, Iguala, Chilpancingo, Teloloapan y Taxco, con el 39,7\% de la población total de Guerrero) presentaron la mayoría de los casos $(64,5 \%)$ en ese año. 
Trece municipios agruparon al $80,1 \%$ de los casos positivos de dengue en 2018 (Tabla 4) y nueve de ellos presentaron una tasa de incidencia por encima de la estatal $(22,4)$. De entre estos municipios destacan Igualapa, Teloloapan e Iguala de la Independencia, que presentaron las tasas de incidencia de dengue más altas en Guerrero $(87,9 \%, 80,2 \%$ y $66,6 \%$, respectivamente), aunque se trata de municipios con poca población, que en conjunto acumulan al 6,2\% de la población de Guerrero.

Figura 3. Distribución municipal de los casos confirmados de dengue en el estado de Guerrero, México, en el año 2018

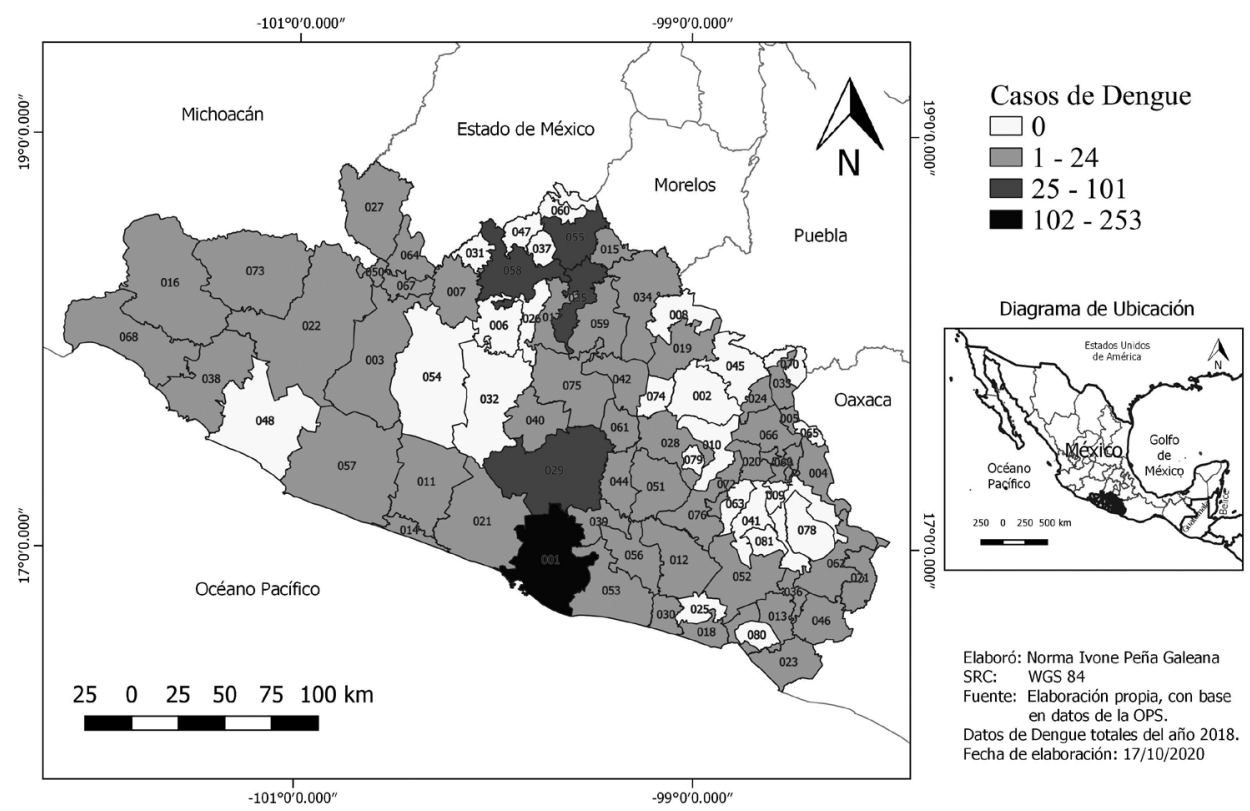

Fuente: Elaboración propia con base en la información publicada por la OPS (n.d.) 
Norma Ivone Peña-Galeana, Ricardo Peña-Galeana, Pedro Vidal Tello-Almague

Vulnerabilidad de la población de Guerrero, México, ante las epidemias de COVID-19 y dengue

Tabla 4. Municipios del estado de Guerrero con mayor cantidad de casos confirmados de dengue en 2018

\begin{tabular}{|c|c|c|c|}
\hline Municipio & Casos confirmados & $\begin{array}{c}\text { Tasa de Incidencia } \\
\text { (Casos por cada 100 mil } \\
\text { habitantes) }\end{array}$ & $\begin{array}{c}\text { \%o de la población } \\
\text { de Guerrero }\end{array}$ \\
\hline Acapulco de Juárez & 253 & 31,2 & $22,9 \%$ \\
\hline Iguala de la Independencia & 101 & 66,6 & $4,3 \%$ \\
\hline Chilpancingo de los Bravo & 68 & 24,9 & $7,7 \%$ \\
\hline Teloloapan & 46 & 80,2 & $3,6 \%$ \\
\hline Taxco de Alarcón & 43 & 39,7 & $1,9 \%$ \\
\hline Ometepec & 24 & 35,5 & $3,1 \%$ \\
\hline Coyuca de Catalán & 20 & 51,3 & $1,4 \%$ \\
\hline Zihuatanejo de Azueta & 16 & 12,8 & $2,0 \%$ \\
\hline San Marcos & 14 & 28,3 & $1,7 \%$ \\
\hline Ayutla de los Libres & 14 & 21,1 & $2,2 \%$ \\
\hline Atoyac de Álvarez & 13 & 15,7 & $0,3 \%$ \\
\hline Coyuca de Benítez & 12 & 87,9 & \\
\hline Igualapa & 10 & & \\
\hline
\end{tabular}

Fuente: Elaboración propia con base en la información publicada por la OPS (n.d.)

Al realizar un análisis conjunto de los casos positivo/confirmados de COVID-19 (al 28 de mayo de 2020) y de dengue (2018) en los municipios de Guerrero, se obtiene una estructura que se muestra en la Figura 4. 
Figura 4. Panorama conjunto de los casos positivos de COVID-19 (2020) y casos confirmados de dengue (2018) en los municipios del estado de Guerrero

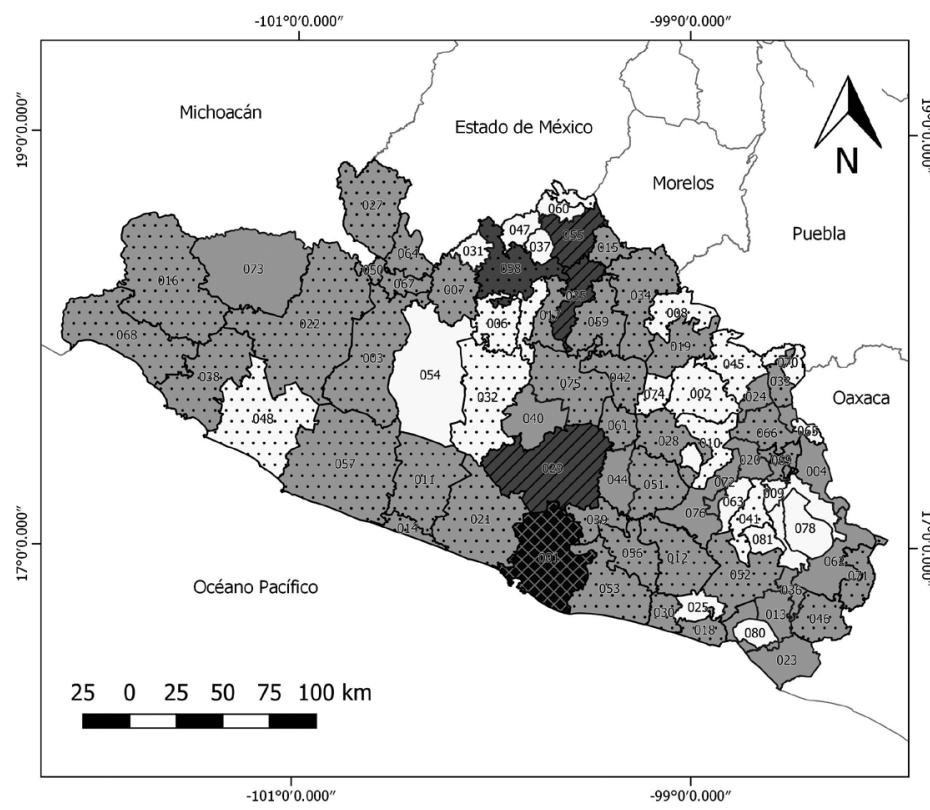

Casos de COVID-19

$\square 0$

四 $1-28$

ए72 $29-147$

$148-991$

Casos de Dengue

$\square 0$

$\square 1-24$

$25-101$

$102-253$

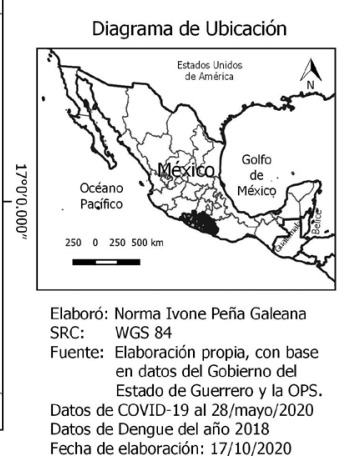

Fuente: Elaboración propia, con base en las Figuras 2 y 3.

Como puede observarse, 13 de los 81 municipios que conforman al estado de Guerrero (con el 4,7\% de la población estatal) no presentaron casos de dengue o COVID-19, 13 municipios (con el 7,5\% de la población) presentaron la menor cantidad de casos en el estado solo de COVID-19 $(2,0 \%)$ y 14 municipios (con el 7,0\% de la población) presentaron una cantidad menor solo de dengue (5,4\%). Lo anterior se traduce en que el 19,3\% de la población estatal, residente en 40 de los municipios, está expuesta a menos fuentes de contagio de alguno de estos dos padecimientos, que el resto de los guerrerenses.

Por el contrario, cuatro municipios acumularon la mayor cantidad de casos, tanto de COVID-19 (82,1\%) como de dengue (58,7\%): Acapulco, Chilpancingo, Iguala y Taxco. Lo anterior es importante porque la población de estos cuatro municipios $(38,0 \%$ de la población de Guerrero) se enfrenta a una mayor posibilidad de contraer alguna de estas dos enfermedades virales, lo cual enfatiza la necesidad de aplicar medidas de 
prevención más agresivas, o de lo contrario la temporada de dengue del 2020 enfrentará a esa población con una emergencia sanitaria aún mayor.

La Figura 5, muestra la relación de la tasa de incidencia (casos por cada 100000 habitantes) de ambos padecimientos, en cada municipio.

Figura 5. Panorama conjunto de la incidencia de COVID-19 y de dengue los municipios del estado de Guerrero
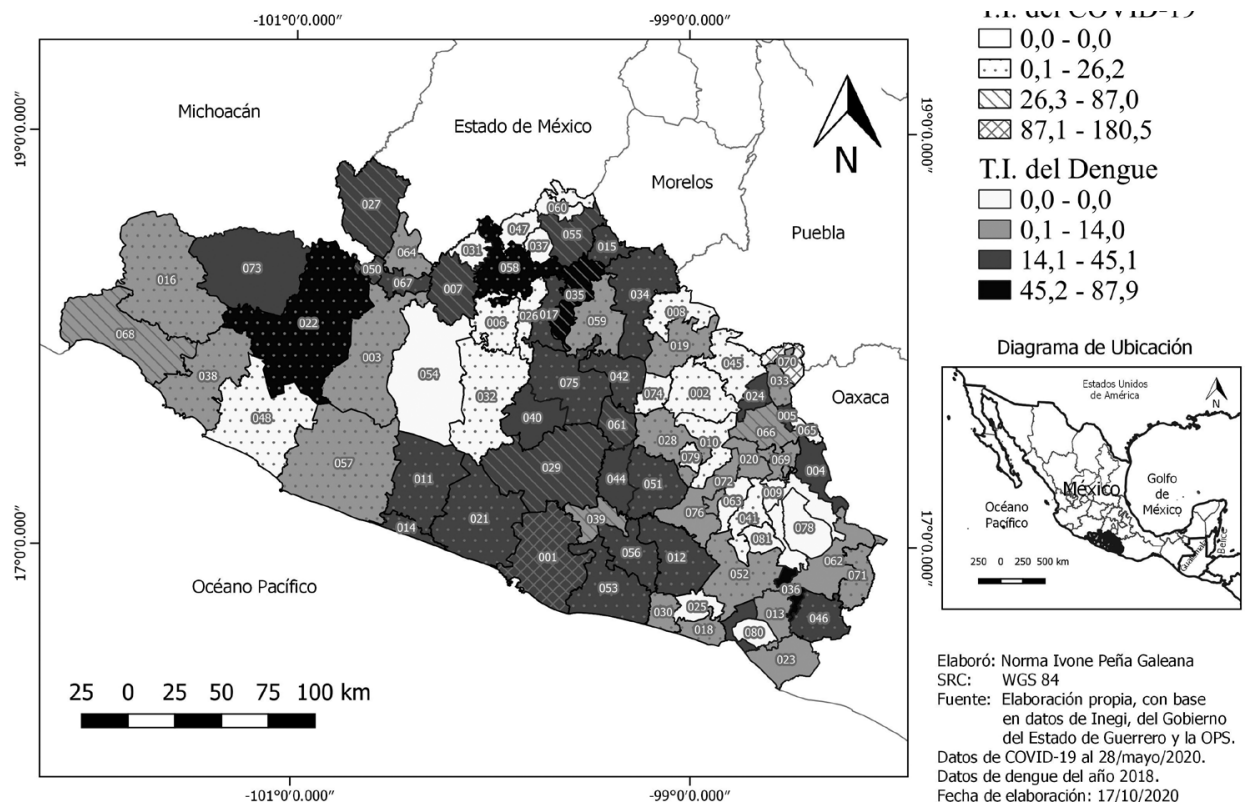

Fuente: Elaboración propia, con base en datos de la OPS (n.d.), del Gobierno del estado de Guerrero (2020b) y del Inegi (2016).

$\mathrm{Al}$ respecto, 45 municipios registraron una tasa de incidencia nula o baja tanto de COVID-19 como de dengue, 30 municipios registraron una incidencia media y solo seis municipios (Iguala, Xochihuehuetlán, Igualapa, Acapulco, Teloloapan y Coyuca de Catalán) registraron tasas de incidencia altas en alguno o en ambos padecimientos. De este último grupo, cabe mencionar lo siguiente:

- $\quad$ Las tasas de incidencia de COVID-19 y dengue en Iguala se encuentran entre las más altas de Guerrero, haciendo que este municipio ocupe el tercer lugar en ambos padecimientos. 
- $\quad$ El municipio de Xochihuehuetlán no registró casos de dengue, pero tiene la incidencia más alta de COVID-19; Igualapa, por el contrario, no registró casos de COVID-19, pero tiene la incidencia más alta de dengue.

- Aunque Acapulco ocupa el primer lugar por el número de casos de ambos padecimientos, en relación con la tasa de incidencia ocupa el tercer lugar en COVID-19 y el lugar 12 en dengue.

\section{Grupos poblacionales en riesgo según edad y sexo}

En relación con el COVID-19, la OMS identifica como principal grupo de riesgo a los adultos mayores y personas con padecimientos crónicos preexistentes, quienes presentan una mayor probabilidad de desarrollar en forma grave este padecimiento. En cuanto al dengue, el mismo organismo define a los niños y adultos mayores como los grupos poblacionales más afectados por este padecimiento, pues se considera que permanecen cerca del domicilio donde se localizan los criaderos artificiales del mosquito transmisor del virus, tales como aljibes, tanques, contenedores de agua o cualquier recipiente sin cubierta que puede almacenar agua (OMS, 2020c; OMS, 2020e).

$\mathrm{Al}$ analizar la tasa de incidencia de la población afectada por $\mathrm{CO}-$ VID-19 y dengue en Guerrero, en relación con el sexo y los grupos de edad, la Tabla 5 muestra que, aunque las mujeres presentan las tasas de incidencia de dengue más altas en todos los grupos de edad, destacan como principales grupos de riesgo para contraer dengue los menores de 20 años de ambos sexos. En el caso del COVID-19, los hombres mayores de 40 años constituyen el principal grupo de riesgo.

Tabla 5. Tasa de incidencia de COVID-19 y dengue en el estado de Guerrero, por grupos de edad y sexo (casos por cada 100,000 habitantes)

\begin{tabular}{|c|c|c|c|c|c|c|}
\hline \multirow{2}{*}{ GRUPO DE EDAD } & \multicolumn{3}{|c|}{ COVID-19' $^{\mathbf{1}}$} & \multicolumn{3}{c|}{ DENGUE $^{2}$} \\
\cline { 2 - 7 } & TOTAL & HOMBRES & MUJERES & TOTAL & HOMBRES & MUJERES \\
\hline Menores de 20 años & 1,5 & 1,2 & 1,7 & 32,3 & 32,2 & 32,5 \\
\hline De 20 a 39 años & 6,1 & 6,2 & 6,1 & 20,8 & 19,2 & 22,1 \\
\hline De 40 a 59 años & 9,7 & 12,7 & 7,1 & 20,5 & 19,8 & 21,1 \\
\hline De 60 años y más & 8,8 & 10,4 & 7,4 & 8,1 & 6,6 & 9,3 \\
\hline
\end{tabular}

Nota: ${ }^{1}$ Cálculos con base en los casos al 13 de abril de 2020; ${ }^{2}$ Calculadas con base en los casos de 2019. Fuente: Elaboración propia con base en los datos de Inegi (2016), del Gobierno de México (2020b) y de la OPS (n.d.). 
Lo anterior tiene importancia debido a que el fin de la cuarentena y la reapertura de las actividades económicas implicará el movimiento de la población trabajadora, donde se ubican los hombres de más de 40 años, aumentando su probabilidad de contraer COVID-19. Asimismo, el cierre de escuelas y las medidas de sana distancia (bajo la frase de "Quédate en casa") implementadas para el control del COVID-19 aumentan la vulnerabilidad de los menores de 20 años ante el dengue, al mantenerlos cerca de los hogares.

\section{Disponibilidad de agua entubada}

Para el combate al COVID-19, el lavado de manos es junto con el distanciamiento social, una de las principales medidas de prevención aplicadas (OPS, 2020b). Esta medida presupone que los hogares cuentan con agua potable o que sus habitantes tienen acceso a este recurso. Guerrero, sin embargo, está clasificado por la Comisión Nacional del Agua (Conagua) como una de "las entidades federativas con mayores rezagos en cobertura de agua potable" (Conagua, 2014, p 98), pues 15,8\% de la población vive en hogares sin agua potable y 22 de los 81 municipios cuentan con un porcentaje mayor al $25 \%$ de ocupantes en viviendas sin agua potable (Inegi, 2016).

Tal como muestra la Tabla 6, destacan los municipios de Huitzuco, Coyuca de Catalán y Olinalá, por ser los municipios con la mayor cantidad de viviendas $\sin$ agua entubada y el mayor porcentaje de ocupantes en viviendas sin agua entubada.

Tabla 6. Principales municipios según la cantidad y el porcentaje de ocupantes en viviendas sin agua entubada.

\begin{tabular}{|c|c|c|c|c|c|}
\hline $\begin{array}{c}\text { Municipios con mayor } \\
\text { porcentaje de ocupantes en } \\
\text { viviendas sin agua entubada }\end{array}$ & Ocupantes & $\mathbf{\%}$ & $\begin{array}{c}\text { Municipios con mayor } \\
\text { cantidad de ocupantes en } \\
\text { viviendas sin agua entubada }\end{array}$ & Ocupantes & $\%$ \\
\hline Copalillo & 14864 & 64,0 & Acapulco de Juárez & 59091 & 7,3 \\
\hline Cuetzala & 8559 & 55,7 & Chilpancingo & 35619 & 13,0 \\
\hline Olinalá & 25483 & 55,4 & Chilapa & 35458 & 27,3 \\
\hline Huitzuco & 37094 & 39,2 & Iguala & 32013 & 21,1 \\
\hline Xalpatláhuac & 11723 & 38,0 & Ometepec & 19924 & 29,5 \\
\hline Coahuayutla & 12721 & 37,3 & Teloloapan & 18449 & 32,2 \\
\hline Coyuca de Catalán & 39002 & 36,7 & Coyuca de Benítez & 18037 & 23,6 \\
\hline Atenango del Río & 8731 & 35,8 & San Marcos & 15249 & 30,8 \\
\hline Azoyú & 14857 & 34,8 & Huitzuco & 14539 & 39,2 \\
\hline Zitlala & 22721 & 34,6 & Coyuca de Catalán & 14328 & 36,7 \\
\hline General Canuto A. Neri & 5780 & 34,6 & Olinalá & 14105 & 55,4 \\
\hline
\end{tabular}

Fuente: Elaboración propia con base en Inegi (2016). 
En el caso del dengue, las medidas de prevención se enfocan en la eliminación de todos los recipientes que pueden convertirse en nichos para las larvas del mosquito transmisor del virus, y en la fumigación para eliminar a los mosquitos adultos. Lo anterior quiere decir que la falta de agua entubada incrementa la vulnerabilidad de la población Guerrerense ante ambos padecimientos pues significa que, para prevenir el contagio por COVID-19, un $15,8 \%$ de la población del estado tendrá una mayor presión por almacenar agua en recipientes que, sin el debido cuidado, pueden convertirse en criaderos de mosquitos y propiciar la aparición de casos de dengue.

A lo anterior, es necesario añadir la irregularidad del servicio de abasto de agua potable, que impone a la población la necesidad de mantener almacenado este líquido a pesar de habitar una vivienda que dispone de agua entubada, por lo que el porcentaje de población en condición de vulnerabilidad se incrementa. Aún más, si a lo anterior se agrega la necesidad de distanciamiento social y la suspensión de clases presenciales, que implican que la población (especialmente los menores) tiende a permanecer más tiempo en los hogares, esto se traduce en una mayor cantidad de personas expuestas a posibles focos de criadero de mosquito.

Finalmente, si se considera que, de acuerdo con el gobierno del estado de Guerrero, el 77\% de los casos positivos de COVID-19 en la Entidad recibe tratamiento ambulatorio (por lo que los pacientes se quedan en casa mientras transcurre la enfermedad), esta población no solo se enfrenta a un mayor riego de dengue debido al almacenamiento de agua, sino que se encuentra expuesta a la fumigación de espacios exteriores que se realiza para controlar a los mosquitos adultos, dado que el éxito de esta medida exige que la población abra sus casas para facilitar la introducción del insecticida en los hogares. Cabe mencionar que a la fecha no existen estudios acerca del impacto que tendrá la fumigación para el dengue en este tipo de pacientes.

\section{Suficiencia alimentaria}

Según los datos de la Encuesta Intercensal 2015 de Inegi, el 11,5\% de los hogares de Guerrero registraron al menos un menor de 18 años que comió solo una vez al día o dejó de comer todo un día por falta de dinero, y este porcentaje se eleva a $17,3 \%$ en el caso de los mayores de 18 años.

A nivel municipal, seis municipios contabilizaron más de $20 \%$ de hogares con menores de 18 años donde algún menor comió solo una vez 
al día o dejó de comer todo un día; estos seis municipios también aparecen entre los 20 que contabilizaron más de $20 \%$ de hogares donde algún adulto comió solo una vez al día o dejó de comer todo un día.

La Tabla 7, destaca los seis municipios que aparecen en estos dos grupos, donde más de la quinta parte de su población (adultos y menores) resultan especialmente vulnerables al COVID-19 y al dengue, pues deberán de hacer frente a ambas enfermedades en condiciones de insuficiencia alimentaria, lo cual con seguridad repercute en una mayor debilidad de su sistema inmunológico (cabe aclarar que no se encontraron estudios al respecto).

Tabla 7. Municipios con mayor porcentaje de hogares con población en condiciones de insuficiencia alimentaria.

\begin{tabular}{|c|c|c|}
\hline Municipios & $\begin{array}{c}\text { Porcentaje de hogares con población } \\
\text { menor de 18 años, donde algún } \\
\text { menor de 18 comió solo una vez al } \\
\text { día o dejó de comer todo un día }\end{array}$ & $\begin{array}{c}\text { Porcentaje de hogares donde } \\
\text { algún adulto comió solo una } \\
\text { vez al día o dejó de comer todo } \\
\text { un día }\end{array}$ \\
\hline Atlamajalcingo del Monte & 28,2 & 32,3 \\
\hline Tlacoachistlahuaca & 26,0 & 30,0 \\
\hline San Luis Acatlán & 23,0 & 26,0 \\
\hline Ajuchitlán del Progreso & 22,9 & 24,4 \\
\hline San Miguel Totolapan & 21,7 & 22,4 \\
\hline Cochoapa el Grande & 21,0 & 26,9 \\
\hline
\end{tabular}

Fuente: Elaboración propia con base en Inegi (2016).

\section{Afiliación a servicios de salud}

Quince por ciento de la población Guerrerense no se encuentra afiliada a ningún servicio de salud (púbico o privado). Esto ocurre en forma más pronunciada en 10 municipios del estado (Tabla 8), donde uno de cada cinco habitantes no se encuentra afiliado a algún servicio de salud y, por tanto, tendrá mayor dificultad para ser diagnosticado y tratado a tiempo en caso de contagiarse con alguno de estos dos virus. 
Tabla 8. Municipios con mayor porcentaje de población no afiliada a algún servicio de salud (público o privado)

\begin{tabular}{|c|c|}
\hline Municipios & $\begin{array}{c}\text { Porcentaje de población municipal no afiliada a } \\
\text { Servicios de Salud }\end{array}$ \\
\hline Zirándaro & 36,8 \\
\hline Pungarabato & 30,4 \\
\hline Cutzamala de Pinzón & 26,0 \\
\hline Tlacoachistlahuaca & 24,8 \\
\hline Tlapehuala & 24,0 \\
\hline San Miguel Totolapan & 23,1 \\
\hline Coyuca de Catalán & 21,0 \\
\hline General Canuto A. Neri & 20,4 \\
\hline Tlalchapa & 20,2 \\
\hline Ajuchitlán del Progreso & 20,1 \\
\hline
\end{tabular}

Fuente: Elaboración propia con base en Inegi (2016).

De este grupo destacan los municipios de Tlacoachistlahuaca, San Miguel Totolapan y Ajuchitlán, por estar también entre los municipios con mayor insuficiencia alimentaria, lo que se traduce en una quinta parte de su población enfrentando ambas epidemias en condiciones de insuficiencia alimentaria y sin tener cobertura de salud.

\section{Vulnerabilidad de la población guerrerense a las epidemias con- juntas de COVID-19 y Dengue}

Al tomar en consideración los factores antes mencionados en conjunto (porcentaje de población en grupos de edad y sexo considerados de riesgo para COVID-19 y dengue, porcentaje de ocupantes en viviendas sin agua entubada, porcentaje de hogares con adultos y menores que no comieron por falta de dinero y porcentaje de población no afiliada a algún servicio de salud), emerge una estructura municipal, tal como se muestra en la Figura 6, donde se revela la vulnerabilidad de la población Guerrerense ante la acción de ambas epidemias. 
Figura 6. Vulnerabilidad de la población Guerrerense ante las epidemias conjuntas de COVID-19 y dengue, con base en los factores seleccionados

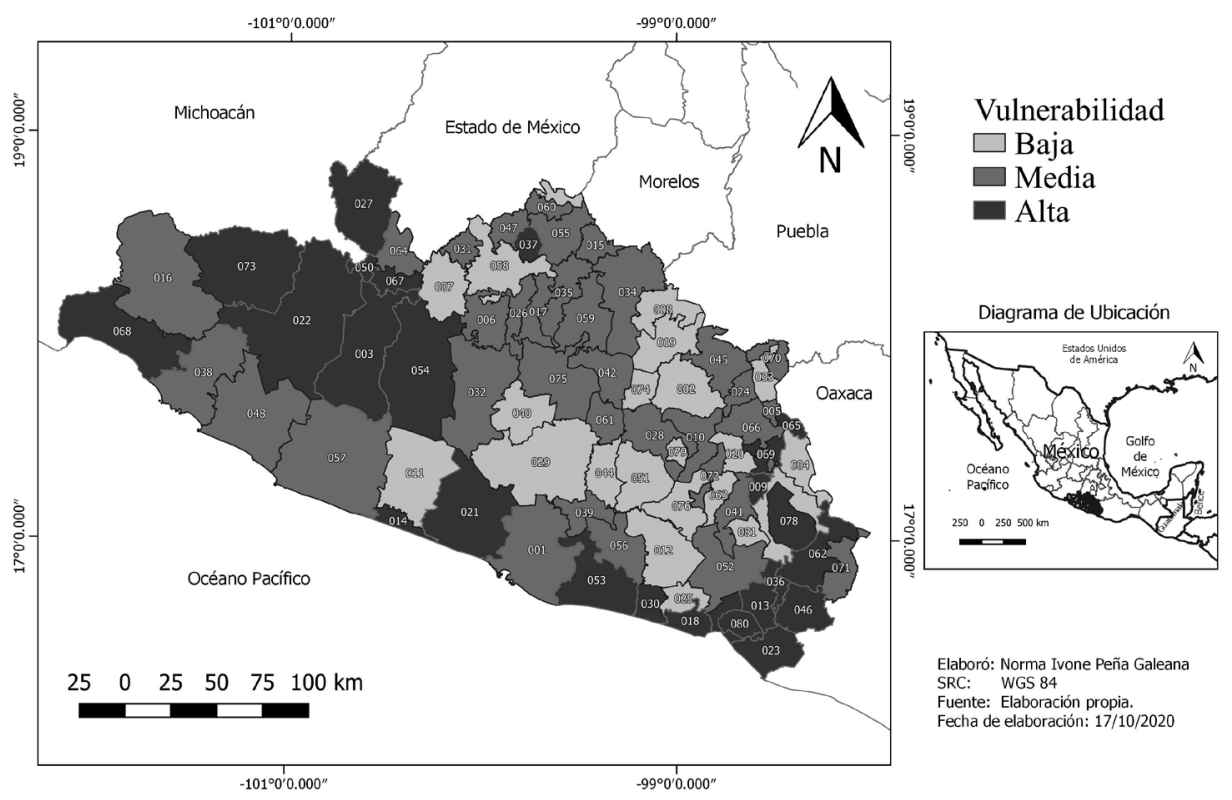

Fuente: Elaboración propia

Como ya se mencionó en el apartado metodológico, los municipios de Guerrero se clasificaron como de baja, media o alta vulnerabilidad con base en un indicador calculado a partir de los valores que asume cada variable en estudio en cada municipio. Con base en esta clasificación, los municipios que aparecen catalogados como de alta vulnerabilidad se definen como aquellos que presentan porcentajes más altos de población en condiciones que los hacen más vulnerables a las epidemias conjuntas de COVID-19 y dengue.

Así, el nivel de vulnerabilidad más alto se encuentra en 22 municipios de Guerrero que acumulan al $17,5 \%$ de la población estatal y que, como puede observarse en la Figura 6, se ubican al noroeste (en la región conocida como la Tierra Caliente) y al sureste de la Entidad (en la región conocida como la Costa Chica).

Esto último es importante, debido a que los municipios de la región Tierra Caliente presentan la mayor proporción de adultos mayores, lo que los hace más susceptibles a desarrollar mayor cantidad de casos graves 
de COVID-19; asimismo, esta conformación implica que, aunque se ha logrado contener el avance del COVID-19 en la Costa Chica, esto puede cambiar con el inicio de la temporada de dengue.

Finalmente, cabe resaltar el caso del Municipio de Acapulco, que se clasifica como de alta vulnerabilidad, donde la atención debe ser prioritaria dado que acumula a más de la quinta parte de la población guerrerense y constituye uno de los principales polos de desarrollo no solo del estado, sino del sur del país.

\section{Discusión}

Uno de los principales puntos de discusión de esta investigación radica en el hecho de que los datos utilizados para el análisis de ambas epidemias corresponden a diferentes periodos de tiempo. Sin embargo, se consideró que el conocimiento de las características de ambos padecimientos y los factores asociados con ellas, reflejaban el panorama general de ambas epidemias y, por tanto, podían utilizarse para analizar la vulnerabilidad de la población derivada de los factores incluidos en el estudio.

Otro aspecto que puede causar controversia, es el cálculo de un indicador de vulnerabilidad a partir de intervalos generados utilizando los valores que asume cada variable en todos los municipios de Guerrero en un periodo de tiempo específico, lo cual hace que dichos intervalos no puedan ser aplicados a otros estados del país ni a otros periodos de tiempo.

Estos intervalos sin embargo, se consideraron válidos debido a que se trató de proporcionar información que sirviera como base para definir estrategias de acción inmediatas en el estado de Guerrero, además de que el procedimiento de creación tanto de los intervalos como del indicador de vulnerabilidad, es fácilmente aplicable a cualquier otro estado en cualquier otro periodo de tiempo.

\section{Conclusiones}

Dada la emergencia de salud a nivel mundial, se considera importante el conocer el nivel de vulnerabilidad de la población ante epidemias emergentes como el COVID-19 y epidemias recurrentes como es el caso del dengue, pues proporcionan una visión más profunda del efecto que éstas pueden tener en la población. Existen, sin embargo, pocos estudios 
de Vulnerabilidad aplicados al estado de Guerrero. Entre los existentes, destacan los trabajos de García y Villerías (2016, 2017a y 2017b), quienes se han abocado al estudio de la vulnerabilidad social en los principales municipios de Guerrero.

La presencia de COVID-19 y dengue en el estado de Guerrero, hace evidente la necesidad de cambios estructurales urgentes en relación con el acceso de la población al agua potable y a los servicios de salud, lo que implica la necesidad de priorizar las inversiones gubernamentales en ambos sectores, especialmente en los municipios denominados como de alta vulnerabilidad.

Además, el inicio de la temporada de dengue y la reanudación de las actividades productivas ocasionará que la población enfrente ambos virus en condiciones de desnutrición, acentuada por la disminución de la actividad económica derivada de la cuarentena por lo que, con el fin de disminuir la vulnerabilidad de los guerrerenses, es necesario realizar acciones que ayuden a la rápida reactivación económica al mismo tiempo que se realicen campañas de educación alimentaria y financiera entre la población.

Asimismo, es urgente el diseño de una estrategia que enfatice los protocolos de prevención del COVID-19 en los centros de trabajo, al mismo tiempo que se intensifican las acciones para evitar la creación de criaderos del mosquito transmisor del dengue en los hogares, escuelas y centros de trabajo. Ante esta situación, también es necesario que se realicen investigaciones acerca del impacto que las acciones de fumigación pueden tener en los pacientes de COVID-19.

Finalmente, en el diseño de cualquier estrategia es necesario tomar en cuenta la relación que guardan las medidas de prevención o control de ambas epidemias, que pueden incidir en el nivel de vulnerabilidad de la población, lo que requiere regionalizar las acciones en función del impacto de las acciones de combate y control de una pandemia sobre la otra.

\section{Referencias}

Araujo, R. (2015). "Vulnerabilidad y riesgo en salud: ¿dos conceptos concomitantes?". Novedades en población, XI (210), pp. 89-96. Recuperado de http://scielo.sld.cu/pdf/rnp/v11n21/rnp070115.pdf

Cardona, O. (2003). "The Need for Rethinking the Concepts of Vulnerability and Risk from a Holistic Perspective: A Necessary Review and Criticism for Effective Risk Management" en G. Bankoff, G. 
Norma Ivone Peña-Galeana, Ricardo Peña-Galeana, Pedro Vidal Tello-Almague Vulnerability of the population of Guerrero state, Mexico, to COVID-19 and dengue epidemics.

Frerks, and D. Hilhorst. (Editors). Mapping vulnerability. Disasters, development and people. Earthscan Publishers, London. Recuperado de https://www.academia.edu/23714845/The_need_for_rethinking the concepts_of_vulnerability_and_risk_from_a holistic

Comisión Económica para América Latina CEPAL-ECLAC (2002). Vulnerabilidad Sociodemográfica: viejos y nuevos riesgos para comunidades, hogares y personas. LC/R.2086. Brasilia, Brasil. Recuperado de https://repositorio.cepal.org/bitstream/handle/11362/13051/ S2002632_es.pdf? sequence $=1 \&$ isAllowed $=y$

Comisión Nacional del Agua de México-Conagua (2014). Estadísticas del agua en México. México, D.F. Recuperado de: http://www.conagua. gob.mx/CONAGUA07/Publicaciones/Publicaciones/EAM2014.pdf Departamento Nacional de Planeación de Colombia-DNP (2007). Una aproximación a la vulnerabilidad. Departamento Nacional de Planeación. Bogotá, Colombia. Recuperado de https://colaboracion. dnp.gov.co/CDT/Desarrollo\%20Social/boletin34_1.pdf

Foschiatti, A. (2008). "El contexto de vulnerabilidad de los procesos demográficos en El Chaco". KAIROS. Revista de Temas Sociales, 12(21). [En línea]. Recuperado de https://www.researchgate.net/publication/28222298_El_contexto_de_vulnerabilidad_de_los_procesos_demograficos_en_El_Chaco

García-Castro, N., \& Villerías-Salinas, S. (2016). "Los niveles de vulnerabilidad social de la ciudad de Chilpancingo, Guerrero, México". Revista Geográfica De América Central, 1(56), pp. 259-288. https:// doi.org/10.15359/rgac.1-56.11

García, N., y Villerías, S. (2017a). "Niveles de vulnerabilidad social del estado de Guerrero, México". [En línea]. Revista Geográfica de Valparaíso, 54, pp. 1-14. Recuperado de: http://158.251.8.152/index. php/revgeo/article/view/13/11

García, N., y Villerías, S. (2017b), "Condiciones de vulnerabilidad social en Ixtapa y Zihuatanejo (Guerrero), México." [En línea]. Revista Geográfica Venezolana, 58(2), pp.264-281. Recuperado de: https:// www.redalyc.org/articulo.oa? $\mathrm{id}=347753793002$

Gobierno de México (2020a). Conferencia Presidente. [Video online] Recuperado de: https://www.youtube.com/watch?v=NNwrYRoK-eo 
GobiernodeMéxico(2020b).InformaciónreferenteacasosCOVID-19enMéxico.Datosabiertos de México [Enlínea]. Recuperadode:https://datos.gob. $\mathrm{mx} /$ busca/dataset/informacion-referente-a-casos-covid-19-en-mexico

Gobierno del Estado de Guerrero (2020a). De los 6 casos sospechosos de Covid-19, cuatro resultaron negativos, uno está pendiente y uno dio positivo, se trata de un ciudadano de origen argentino. Portal oficial del Gobierno del estado de Guerrero. 15 de marzo de 2020. [En línea]. Recuperado de: http://guerrero.gob.mx/2020/03/de-los-6-casos-sospechosos-de-covid-19-cuatro-resultaron-negativos-uno-esta-pendiente-y-uno-dio-positivo-se-trata-de-un-ciudadano-de-origen-argentino/

Gobierno del Estado de Guerrero (2020b). Panorama sobre la situación en Guerrero por el COVID-19. Portal oficial del Gobierno del estado de Guerrero, 28 de mayo de 2020. [En línea]. Recuperado de: http://i.guerrero.gob.mx/uploads/2020/05/Corte-por-municipioo-al28-de-mayo.pdf

Instituto Nacional de Estadística, Geografía e Informática de Mexico-Inegi (2016). Encuesta Intercensal 2015. Tabulados Guerrero. Portal del Inegi [En línea]. Recuperado de: https://www.inegi.org.mx/programas/intercensal/2015/default.html\#Tabulados

Instituto Nacional de Estadística, Geografía e Informática de Mexico-Inegi (2019). Marco geoestadístico, septiembre 2019. Portal del Inegi [En línea]. Recuperado de: https://www.inegi.org.mx/app/mapas/

Organización Mundial de la Salud-OMS (2016). Dengue vaccine: WHO position paper. Weekly epidemiological record. 30(91):349-364. Recuperado de: https://www.who.int/wer/2016/wer9130/en/

Organización Mundial de la Salud-OMS (2018). Dengue vaccine: WHO position paper. Weekly epidemiological record. 36(93):457-476. Recuperado de: https://apps.who.int/iris/bitstream/handle/10665/274315/ WER9336.pdf?ua=1

Organización Mundial de la Salud-OMS (2020a). Statement on the second meeting of the International Health Regulations Emergency Committee regarding the outbreak of novel coronavirus (2019-nCoV). 30 de enero de 2020. [En línea]. Recuperado de: https:/www.who.int/ news/item/30-01-2020-statement-on-the-second-meeting-of-the-international-health-regulations-(2005)-emergency-committee-regarding-the-outbreak-of-novel-coronavirus-(2019-ncov) 
Norma Ivone Peña-Galeana, Ricardo Peña-Galeana, Pedro Vidal Tello-Almague Vulnerability of the population of Guerrero state, Mexico, to COVID-19 and dengue epidemics.

Organización Mundial de la Salud-OMS (2020b). WHO Director-General's opening remarks at the media briefing on COVID-19. 11 de marzo de 2020. [En línea]. Recuperado de: https://www.who.int/dg/speeches/detail/who-director-general-s-opening-remarks-at-the-mediabriefing-on-covid-19---11-march-2020

Organización Mundial de la Salud-OMS (2020c). Dengue y dengue grave. Datos y cifras. Centro de prensa, 2 de marzo de 2020. [En línea]. Recuperado de: https://www.who.int/es/news-room/fact-sheets/detail/ dengue-and-severe-dengue

Organización Mundial de la Salud-OMS (2020d). Lucha contra el Dengue.

[En línea]. Recuperado de: https:/www.who.int/denguecontrol/es/

Organización Mundial de la Salud-OMS (2020e). Preguntas y respuestas sobre la enfermedad por coronavirus (COVID-19). [En línea]. Recuperado de: https://www.who.int/es/emergencies/diseases/novel-coronavirus-2019/ advice-for-public/q-a-coronaviruses

Organización Panamericana de la Salud-OPS (2020a). Control of Aedes aegypti in the scenario of simultaneous transmission of COVID-19. Interim Guidelines. Version 1 [En línea]. Recuperado de: https:// www.paho.org/en/documents/control-aedes-aegypti-scenario-simultaneous-transmission-covid-19

Organización Panamericana de la Salud-OPS (2020b). Recommendations to expand access to hand washing and its proper use. [En línea]. Recuperado de: https://iris.paho.org/bitstream/ handle/10665.2/52064/PAHOCDECECOVID19200013_eng. pdf? sequence $=1 \&$ isAllowed $=y$

Organización Panamericana de la Salud-OPS (n.d.) Casos reportados de Dengue. Plataforma de Información en Salud para las Américas (Plisa) [En línea]. Recuperado de: https://www.paho.org/data/index.php/es/temas/ indicadores-dengue/dengue-subnacional/518-mex-egi-casos-es.html

Porto, C. (2015). Cartografía de América. Orogénesis Soluciones Geográficas. Porlamar, Venezuela. Recuperado de: https://tapiquen-sig.jimdofree.com/descargas-gratuitas/am\%C3\%A9rica/

Saavedra, F. (2010), Vulnerabilidad de la Población Frente a Inundaciones e Inestabilidad de Laderas. In Cotler Ávalos, H. (coord.). Las cuencas hidrográficas de México: diagnóstico y priorización, pp. 132-137. 
México: Semarnap-INE, Fundación Río Arronte. Recuperado de http:// www2.inecc.gob.mx/publicaciones2/libros/639/vulnerabilidad.pdf

Shitangsu, P. (2014). Vulnerability concepts and its application in various fields: a review on geographical perspective. J. Life Earth Sci. 8, pp. 63-81. https://doi.org/10.3329/jles.v8i0.20150

Secretaría de Salud de México-SSA (2003). NOM-032-SSA2-2002 para la vigilancia epidemiológica, prevención y control de las enfermedades transmitidas por vector. Diario Oficial de la Federación, Lunes 21 de julio de 2003. [En línea]. Recuperado de: https://dof.vlex.com. $\mathrm{mx} /$ vid/epidemiologica-transmitidas-vector-28062311

Secretaría de Salud de México-SSA (2015). NOM-032-SSA2-2014 para la vigilancia epidemiológica, prevención y control de las enfermedades transmitidas por vectores. Diario Oficial de la Federación, Jueves 16 de abril de 2015. [En línea]. Recuperado de: http://www.cenaprece.salud.gob.mx/ programas/interior/vectores/descargas/pdf/NOM_032_SSA2_2014.pdf

Secretaría de Salud de México-SSA (2017a). Manual de procedimientos estandarizados para la vigilancia epidemiológica de las enfermedades transmitidas por vector. Recuperado de: https://hospitalcomunitariotarimoro.files.wordpress.com/2017/06/manual-etv-final-final01082016-conave.pdf

Secretaría de Salud de México-SSA (2017b). Panorama Epidemiológico de Dengue, 2016. Portal del Gobierno de México [En línea]. Recuperado de: https://www.gob.mx/cms/uploads/attachment/file/178952/ Pano dengue sem 52 2016.pdf

Secretaría de Salud de México-SSA (2018). Panorama Epidemiológico de Dengue, 2017. Portal del Gobierno de México [En línea]. Recuperado de: https://www.gob.mx/cms/uploads/attachment/file/285237/ Pano_dengue_sem_52_2017.pdf

Secretaría de Salud de México-SSA (2019). Panorama Epidemiológico de Dengue, 2018. Portal del Gobierno de México [En línea]. Recuperado de: https://www.gob.mx/cms/uploads/attachment/file/478592/ Pano_dengue_sem_52_2018.pdf

Secretaría de Salud de México-SSA (2020). Panorama Epidemiológico de Dengue, 2019. Portal del Gobierno de México [En línea]. Recuperado de: https://www.gob.mx/cms/uploads/attachment/file/524262/ Pano_dengue_52_2019.pdf 
